\title{
New $\Phi$ BT1 site-specific integrative vectors with neutral phenotype in Streptomyces
}

\author{
Nathaly Gonzalez-Quiñonez ${ }^{1}$ - María Teresa López-García ${ }^{1}$ Paula Yagüe ${ }^{1}$. \\ Beatriz Rioseras $^{1} \cdot$ Annalisa Pisciotta $^{2} \cdot$ Rosa Alduina $^{2} \cdot$ Ángel Manteca ${ }^{1}$
}

Received: 29 September 2015 / Revised: 15 December 2015 / Accepted: 19 December 2015 /Published online: 13 January 2016

(C) Springer-Verlag Berlin Heidelberg 2016

\begin{abstract}
Integrative plasmids are one of the best options to introduce genes in low copy and in a stable form into bacteria. The $\Phi C 31$-derived plasmids constitute the most common integrative vectors used in Streptomyces. They integrate at different positions ( $a t t B$ and pseudo-attB sites) generating different mutations. The less common $\Phi B T 1-$ derived vectors integrate at the unique attB site localized in the $S C O 4848$ gene ( $S$. coelicolor genome) or their orthologues in other streptomycetes. This work demonstrates that disruption of SCO4848 generates a delay in spore germination. SCO4848 is cotranscribed with $S C O 4849$, and the spore germination phenotype is complemented by $S C O 4849$. Plasmids pNG1-4 were created by modifying the $\Phi \mathrm{BT} 1$ integrative vector pMS 82 by introducing a copy of SCO4849 under the control of the promoter region of SCO4848. pNG2 and pNG4 also included a copy of the $P_{\text {erme }} *$ in order to facilitate gene overexpression. pNG3 and pNG4 harboured a copy of the bla gene (ampicillin resistance) to facilitate selection in E. coli. pNG1-4 are the only integrative vectors designed to produce a neutral phenotype when they are integrated into the Streptomyces genome. The experimental approach developed in this work can be
\end{abstract}

Electronic supplementary material The online version of this article (doi:10.1007/s00253-015-7271-0) contains supplementary material, which is available to authorized users.

Ángel Manteca

mantecaangel@uniovi.es

1 Área de Microbiología, Departamento de Biología Funcional e IUOPA, Facultad de Medicina, Universidad de Oviedo, 33006 Oviedo, Spain

2 Department of Biological, Chemical and Pharmaceutical Sciences and Technologies, University of Palermo, Palermo, Italy applied to create phenotypically neutral integrative plasmids in other bacteria.

Keywords Streptomyces . $\Phi \mathrm{BT} 1$ integrative vector . Heterologous expression $\cdot$ Neutral phenotype

\section{Introduction}

Streptomycetes are important industrial bacteria, which produce two thirds of all clinically relevant secondary metabolites (Hopwood 2007; Davies 2014). They have complex life cycles that include sporulation and developmentally associated programmed cell death, and they are considered multicellular prokaryotic models (reviewed in Yagüe et al. 2013).

Streptomyces genetic methodologies have been extensively optimized during the last decades (Kieser et al. 2000). Vectors including the $\Phi \mathrm{C} 31 \mathrm{attP}$ site were the first and the most commonly used integrative plasmids designed to work in Streptomyces (Bierman et al. 1992). The major limitation of these types of vectors is that they integrate at different positions in the Streptomyces genome, resulting in different mutations and phenotypes (Combes et al. 2002). This issue is especially relevant when $\Phi C 31$ plasmids are used to introduce genes in Streptomyces mutants in order to complement their mutations: phenotypes can be a consequence of the integration of the plasmid instead to the function of the gene that is being harboured by the plasmid.

The alternatives to the $\Phi \mathrm{C} 31$ integrative site in Streptomyces are the BT1 (Gregory et al. 2003), TG1 (Morita et al. 2009) and SV1 (Fayed et al. 2014) integrative sites. The $\Phi B T 1$ derived vectors integrate into the attP site localized into the SCO4848 gene (S. coelicolor genome) (Gregory et al. 2003), while the TG1 plasmids integrate at SCO3658 (Fayed et al. 2014) and SV1 plasmids at SCO4383 (Fayed et al. 2014). To 
our knowledge, there are no reports describing the phenotype generated by the integration of TG1- and SV1-derived plasmids. However, in the case of $\Phi \mathrm{BT} 1$ plasmids, it was reported that integration into $S C O 4848$ leads to the expression of a modified but functional version of SCO4848, generating a neutral phenotype once they are integrated (Gregory et al. 2003).

In this work, a detailed phenotypic analysis of $S$. coelicolor harbouring the $\Phi B T 1$ integrative vector pMS82 (Gregory et al. 2003) resulted in the identification of a novel phenotype consisting of a delay in spore germination. It was demonstrated that the disruption of SCO4848 affected the expression of SCO4849, and the detected phenotype is complemented by SCO4849. SCO4848 and SCO4849 encode for putative transmembrane proteins with unknown functions which are highly conserved in streptomycetes.

Four plasmids were created modifying the $\Phi \mathrm{BT} 1$ integrative vector pMS82 (Gregory et al. 2003) in order to restore SCO4849 expression: pNG1, in which the SCO4849 gene was introduced under the control of the promoter region of SCO4848 into pMS82 to complement the phenotype generated by the integration of the plasmid; pNG2, in which the $P_{\text {erme }}{ }^{*}$ promoter was introduced into pNG1 to facilitate heterologous gene expression; pNG3, in which the bla gene for ampicillin resistance was included in pNG1 to facilitate selection in E. coli; and pNG4, in which the bla gene was introduced into pNG2. As discussed below, these plasmids are the only integrative vectors designed to produce a neutral phenotype when they are integrated in Streptomyces.

\section{Materials and methods}

\section{Bacterial strains and media}

Streptomyces coelicolor M145, S. griseus IFO 13350, S. clavuligerus ATCC 27064 and S. lividans 1326 were the reference strains used in this work (Table 1). Petri dishes $(8.5 \mathrm{~cm})$ with $25 \mathrm{ml}$ of solid medium were covered with cellophane discs (cellulose acetate films were from Sadipal), inoculated with $100 \mu \mathrm{l}$ of a spore suspension $\left(1 \times 10^{8}\right.$ viable spores $/ \mathrm{ml}$ ), and incubated at $30{ }^{\circ} \mathrm{C}$. Glucose, yeast $/ \mathrm{malt}$ (GYM) extract (Novella et al. 1992) was the medium used for $S$. coelicolor, S. griseus and S. lividans cultures. Streptomyces clavuligerus germinates very slowly in GYM, and for this reason, Trypticase soy agar (TSA) (Kieser et al. 2000) was used for spore germination of this strain. Unless otherwise stated, soya flour mannitol (SFM) (Kieser et al. 2000 ) was the medium used to obtain spores of $S$. coelicolor, S. lividans and S. griseus. Streptomyces clavuligerus do not sporulate well in SFM, and therefore, MA (Sánchez and Braña 1996) was used for sporulation of this strain.
Liquid cultures were performed in R5A sucrose-free liquid medium (Fernandez et al. 1998). Laboratory flasks of $500 \mathrm{ml}$ were filled with $100 \mathrm{ml}$ of culture medium, inoculated with $1 \times 10^{7}$ spores $/ \mathrm{ml}$ and incubated at $200 \mathrm{rpm}$ and $30^{\circ} \mathrm{C}$.

The influence of the medium used to raise the spores was tested in S. coelicolor, comparing germination of spores obtained in SFM or GYM. The effect of the medium used for germination was analysed testing germination of all the strains in two additional media: MM with glucose and R2YE (Kieser et al. 2000).

E. coli strains were grown at $37{ }^{\circ} \mathrm{C}$ in solid $(2 \%$ agar $)$ or liquid 2xYT medium (Sambrook and Russell 2001) supplemented with the appropriate antibiotics (Table 1): ampicillin was used at $100 \mu \mathrm{g} / \mathrm{ml}$, kanamycin at $50 \mu \mathrm{g} / \mathrm{ml}$ and hygromycin at $100 \mu \mathrm{g} / \mathrm{ml}$. The antibiotic used for selection in Streptomyces was hygromycin at the final concentration of $200 \mu \mathrm{g} / \mathrm{ml}$.

\section{Viability staining}

Culture samples were obtained and processed for microscopy at different incubation time points, as previously described (Manteca et al. 2006). Cells were stained with propidium iodide and SYTO 9 (LIVE/DEAD BacLight Bacterial Viability Kit, Invitrogen, L-13152). The samples were observed under a Leica TCS-SP2-AOBS confocal laser scanning microscope at wavelengths of 488 and $568 \mathrm{~nm}$ excitation and $530 \mathrm{~nm}$ (green) or $640 \mathrm{~nm}$ (red) emissions (Manteca et al. 2006).

\section{Spore germination}

Germination was quantified as previously reported (de Jong et al. 2009). Briefly, germination was quantified in solid media with cellophane discs. At different developmental time points, pieces of cellophane discs were cut and processed for confocal microscopy as described in the previous paragraph. Three biological replicates of the cultures were analysed at different developmental time points. The percentage of germination was assessed from at least 100 spores at each time point. Spores were considered to be germinating when the germ tubes were visible under the confocal microscope.

\section{Antibiotic quantification}

Undecylprodigiosin and actinorhodin were quantified spectrophotometrically according to Tsao et al. (1985) and Bystrykh et al. (1996). In order to measure the total amount of actinorhodin (intracellular and extracellular), cells were ruptured in their culture medium by adding $\mathrm{KOH} 0.1 \mathrm{~N}$. Cellular debris was discarded by centrifugation, and actinorhodin was quantified spectrophotometrically with a UV/visible spectrophotometer (Shimadzu, Model UV-1240), applying the linear Beer-Lambert relationship to estimate concentration 
Table 1 Bacterial strains, plasmids and primers used in this study

\begin{tabular}{|c|c|c|}
\hline Strain, plasmid, cosmid & Description & Origin or reference \\
\hline \multicolumn{3}{|l|}{ Bacterial strains } \\
\hline S.coelicolor M145 & $\mathrm{SCP} 1^{-} \mathrm{SCP} 2^{-}$ & Kieser et al. (2000) \\
\hline S. griseus & IFO 13350 & IFO \\
\hline S. clavuligerus & ATCC 27064 & ATCC \\
\hline S. lividans & 1326 & Kieser et al. (2000) \\
\hline$\Delta \mathrm{SCO0995}$ & S. coelicolor SCO0995 mutant: SCO0995::Tn5062, Am ${ }^{\mathrm{R}}$ & This study \\
\hline E. coli $\mathrm{TOP} 10$ & $\begin{array}{l}\text { F- mcrA } \Delta(\text { mrr-hsdRMS-mcrBC) } \varphi 801 \text { acZ } \Delta \text { M15 } \Delta \text { lacX74 } \\
\text { nupG recA1 araD139 } \Delta \text { (ara-leu)7697 galE15 galK16 rpsL } \\
\left(\mathrm{Str}^{\mathrm{R}}\right) \text { endA1 } \lambda^{-}\end{array}$ & Invitrogen \\
\hline E. coli $\mathrm{ET} 12567$ & dam-13::Tn9, dem-6 & MacNeil et al. (1992) \\
\hline E. coli ET12567/pUZ8002 & $\begin{array}{l}\text { E. coli } \mathrm{ET} 12567 \text { containing plasmid pUZ8002, a not self- } \\
\text { transmissible plasmid which can mobilize other plasmids }\end{array}$ & Flett et al. (1997) \\
\hline \multicolumn{3}{|l|}{ Plasmids and cosmids } \\
\hline pCR $^{\text {TM}}$-Blunt II-TOPO ${ }^{\circledR}$ & Zero Blunt $^{\circledR}$ TOPO $^{\circledR}$ PCR Cloning Kit, $\mathrm{Km}^{\mathrm{R}}$ & Invitrogen \\
\hline pQM5062 & Plasmid containing $e G F P$ Tn5062 & Bishop et al. (2004) \\
\hline pTOPO-P $\mathrm{SCO}_{\mathrm{S} 48}$ & $\mathrm{P}_{\mathrm{SCO} 4848}$ cloned in Zero Blunt ${ }^{\circledR} \mathrm{TOPO}^{\circledR}, \mathrm{Km}^{\mathrm{R}}$ & This study \\
\hline pTOPO-SCO4849 & SCO4849 cloned in Zero Blunt ${ }^{\mathbb{R}} \mathrm{TOPO}^{\circledR}, \mathrm{Km}^{\mathrm{R}}$ & This study \\
\hline pTOPO-P $\mathrm{SCO}_{848}+\mathrm{SCO} 4849$ & $\mathrm{P}_{\mathrm{SCO} 4848} \mathrm{SCO} 4849$ cloned in Zero Blunt ${ }^{\circledR} \mathrm{TOPO}^{\circledR}, \mathrm{Km}^{\mathrm{R}}$ & This study \\
\hline pMS82 & Cloning vector, $\mathrm{Hyg}^{\mathrm{R}}$ & Gregory et al. (2003) \\
\hline pNG1 & $\mathrm{P}_{\mathrm{SCO} 4848}+\mathrm{SCO} 4849$ cloned into pMS82/NsiI/HindIII, Hyg ${ }^{\mathrm{R}}$ & This study; accession KR131848 \\
\hline pNG1', & pNG1 without the $N d e I$ cutting site, Hyg $^{\mathrm{R}}$ & This study \\
\hline pNG2 & $P_{\text {ermE }}{ }^{*}+\mathrm{RBS}+\mathrm{MCS}+$ fd-ter cloned into $\mathrm{pNG1} 1^{\prime} /$ EcoRV/SpeI Hyg ${ }^{\mathrm{R}}$ & This study; accession KR131849 \\
\hline pNG3 & bla cloned into pNG1/HindIII/AvrII Hyg ${ }^{\mathrm{R}}, \mathrm{Amp}^{\mathrm{R}}$ & This study; accession KR131850 \\
\hline pNG4 & bla cloned into pNG2/HindIII/AvrII Hyg ${ }^{\mathrm{R}}, \mathrm{Amp}^{\mathrm{R}}$ & This study; accession KR131851 \\
\hline $\begin{array}{l}\text { 2StG2.1.G12 } \\
\text { pNG2-SCO0995 }\end{array}$ & $\begin{array}{l}\text { StG2 cosmid carrying Tn5062 transposon interrupting SCO0995 ORF } \\
\text { pNG2 plasmid containing the ORF of SCO0995 under the control of } P_{\text {ermE }}{ }^{*}\end{array}$ & Fernández-Martínez et al. (2011) \\
\hline \multicolumn{3}{|c|}{ F } \\
\hline PSCO4848F & 5' GGAAGCTTGCGAACAGCATCTTCAGGG 3' & This study \\
\hline PSCO4848R & 5' AACATATGGGTTTCAGTATCGCCGCAC 3' & This study \\
\hline SCO4849F & 5' GGCATATGGCGACGAGGACGTCACGGA 3' & This study \\
\hline SCO4849R & 5' AAATGCATGGGGATGACGAAGGTGATG 3' & This study \\
\hline Bla-F & 5' AAACCTAGGAAATATGTATCCGCTCATG 3' & This study \\
\hline Bla-R & 5' GGGAAGCTTAGGGATTTTGGTCATGAG 3' & This study \\
\hline $\mathrm{SCO} 4848 \mathrm{~F}$ & 5' CGTCGTATCCCCTCGGTTG 3' & This study \\
\hline pMS82R & 5' GAGCCGGGAAAGCTCATTCA 3' & This study \\
\hline SCO0995F & 5' CATATGGCACGAACGGGCGGGTT 3' & This study \\
\hline SCO0995R & 5' ACTAGTATGACCCGCTGATACTACGG 3' & This study \\
\hline RT4848F & 5' GGATCCGGACAGCGTGCG 3' & This study \\
\hline RT4849F & 5'ATGGTGGTCGTCTTCGTGCT 3' & This study \\
\hline RT4849R & 5' CAGCGGCCGGACGACCT 3' & This study \\
\hline RT0995F & 5' GAACTGCTCGACACCTCTCC 3' & This study \\
\hline RT0995R & 5' GTCTGCACCATCAGGTCCTC 3' & This study \\
\hline
\end{tabular}

$(\mathrm{e} 640=25,320)$. Undecylprodigiosin was measured after vacuum drying the culture (including the mycelium and culture medium) followed by extraction with methanol, acidification with $\mathrm{HCl}$ (to $0.5 \mathrm{M}$ ) and spectrophotometric assay at $530 \mathrm{~nm}$, again using the Beer-Lambert relationship to estimate concentration $(\mathrm{e} 530=100,500)$. In all cases, for the high concentration solutions, dilutions were performed to conduct the analysis in the linear Beer-Lambert region.

Protein quantification Samples (half millilitre) were collected at different developmental time points, and stored at $-20^{\circ} \mathrm{C}$ until they were analysed. Half millilitre of $\mathrm{NaOH} 1 \mathrm{M}$ was 
added to the half millilitre samples $(0.5 \mathrm{M}$ final concentration of $\mathrm{NaOH}$ ) and boiled for $10 \mathrm{~min}$. Cellular debris was removed by centrifugation (at $7740 \times g$ for $15 \mathrm{~min}$ at $4{ }^{\circ} \mathrm{C}$ ), in order to obtain the intracellular samples. Protein was quantified using the Bradford method (Bradford 1976) with bovine serum albumin (Sigma) as the standard.

\section{Confirmation of the $\Phi B T 1$ integration site}

Two primers were designed that hybridize to the SCO4848 5' end (SCO4848F, hybridizing 33 bp upstream of the SCO4848 $\mathrm{ORF}$ ) and the DNA region flanking attP in pMS82 (pMS82R, hybridizing at $368 \mathrm{bp}$ downstream of the pMS82 attP). These primers amplify a fragment of $617 \mathrm{bp}$ only if pMS82 or their derived plasmids (pNG1-4) are integrated at the $a t t B$ site of SCO4848.

\section{RT-PCR}

Total RNA samples were extracted from 24- and 72-h cultures of $S$. coelicolor growing in GYM solid media with cellophane. RNA samples were obtained using phenol extraction and the RNeasy Midi Kit (Qiagen) and chromosomal DNA was removed after DNase I (Qiagen) and Turbo DNase (Ambion) treatments. RNA integrity was verified by means of the 2100 Bioanalyzer (Agilent). Reverse transcription (RT)-PCR analysis was performed using the SuperScript one-step RT-PCR system with Platinum Taq DNA polymerase (Invitrogen), using $200 \mathrm{ng}$ of total RNA as template. Positive controls were performed using chromosomal DNA as template.

RNA obtained at $72 \mathrm{~h}$ and primers RT4848F/RT4849R (hybridizing at position -15 of SCO4848 ORF and 285 of SCO4849 ORF, respectively) were used for the analysis of SCO4848 and SCO4849 co-transcription (623-bp amplicon). Reverse transcription was performed at $55^{\circ} \mathrm{C}$ for $30 \mathrm{~min}$ followed by an initial denaturation at $94^{\circ} \mathrm{C}$ for $2 \mathrm{~min}$. Then, a touchdown was performed in 10 cycles during which the annealing temperature was reduced by $1{ }^{\circ} \mathrm{C}$ in each cycle: $94{ }^{\circ} \mathrm{C}$ for $15 \mathrm{~s}, 65^{\circ} \mathrm{C}\left(\Delta-1{ }^{\circ} \mathrm{C}\right)$ for $30 \mathrm{~s}$ and $68^{\circ} \mathrm{C}$ for $35 \mathrm{~s}$. For the next 35 cycles $\left(94^{\circ} \mathrm{C}\right.$ for $15 \mathrm{~s}, 55^{\circ} \mathrm{C}$ for $30 \mathrm{~s}$ and $68^{\circ} \mathrm{C}$ for $35 \mathrm{~s}$ ), the annealing temperature was set at $55^{\circ} \mathrm{C}$ with a final extension step at $68{ }^{\circ} \mathrm{C}$ for $5 \mathrm{~min}$.

RNA obtained at $24 \mathrm{~h}$ and primers RT0995F/RT0995R were used for the analysis of SCO0995 gene expression (420-bp amplicon). First-strand complementary DNA (cDNA) synthesis was performed at $50^{\circ} \mathrm{C}$ for $30 \mathrm{~min}$ followed by an initial denaturation at $94{ }^{\circ} \mathrm{C}$ for $2 \mathrm{~min}$. Then, a touchdown was performed in 6 cycles with a $1{ }^{\circ} \mathrm{C}$ reduction in the annealing temperature in each cycle: $94{ }^{\circ} \mathrm{C}$ for $15 \mathrm{~s}$, $66{ }^{\circ} \mathrm{C}\left(\Delta-1{ }^{\circ} \mathrm{C}\right)$ for 30 s and $68{ }^{\circ} \mathrm{C}$ for $45 \mathrm{~s}$. An annealing temperature of $60^{\circ} \mathrm{C}$ was used in the next 35 cycles $\left(94^{\circ} \mathrm{C}\right.$ for $15 \mathrm{~s}, 55^{\circ} \mathrm{C}$ for $30 \mathrm{~s}$ and $68^{\circ} \mathrm{C}$ for $45 \mathrm{~s}$ ) with a final extension step at $68{ }^{\circ} \mathrm{C}$ for $5 \mathrm{~min}$.
Fig. 1 Effect of pMS82 in spore germination and integration in the S. coelicolor chromosome. a Percentage of germination in S. coelicolor M145 wild-type strain and S. coelicolor harbouring pMS82 growing in GYM medium. Germination values correspond to the average \pm SD of three biological replicates. b Confocal microscope images of $S$. coelicolor and $S$. coelicolor pMS82 cultures at 4 and $7 \mathrm{~h}$. Arrows point to ungerminated spores. $\mathbf{c}$ Scheme of the integration of pMS82 into the S. coelicolor chromosome. Primers SCO4848F and pMS82R are indicated. Int 1 and int 2 indicate the mutated versions of SCO 4848 generated by the integration of the plasmid. d PCR confirming the integration of pMS82 into the SCO4848 attB site. e Comparison of the amino acid sequences of SCO4848 and their mutated versions (int 1 and int2). f Scheme of the chromosomal region around SCO4848 illustrating the position of the primers RT4848F and RT4849R. g RT-PCR with primers RT4848F and RT4849R demonstrating the co-transcription of SCO4848 and SCO4849 (RNA lane); the DNA lane corresponds to PCR using chromosomal DNA as template (positive control). $M$ molecular weight marker. $\mathbf{h}$ Scheme of the chromosomal region around SCO4848 illustrating the position of the primers RT4849F and RT4849R. i RT-PCR illustrating the alteration of the transcription of SCO4849 in the S. coelicolor strain harbouring pMS82

RNA obtained at $24 \mathrm{~h}$ and primers RT4849F/RT4849R (hybridizing at position 1 and 285 of SCO4849) were used for the analysis of SCO4849 transcription (297-bp amplicon). RT-PCR conditions were as follows: first-strand cDNA synthesis was performed at $60^{\circ} \mathrm{C}$ for $30 \mathrm{~min}$ and denaturation at $94{ }^{\circ} \mathrm{C}$ for $2 \mathrm{~min}$. PCR amplification in 40 cycles of $94{ }^{\circ} \mathrm{C}$ for $15 \mathrm{~s}, 65^{\circ} \mathrm{C}$ for $30 \mathrm{~s}$ and $68^{\circ} \mathrm{C}$ for $15 \mathrm{~s}$ and final extension at $68{ }^{\circ} \mathrm{C}$ for $5 \mathrm{~min}$. This RT-PCR generated unspecific amplicons, but the identity of the 297-bp amplicon was confirmed by Sanger DNA sequencing.

\section{Gene synthesis}

The synthesis of SCO4848 including silent mutations in the attB site (Fig. 1d) (accession number KR150757) and $P_{\text {ermE }}{ }^{*+}$ RBS+MCS+fd-ter (accession number KR131846) were ordered from GeneCust Europe.

\section{pNG1 construction}

The fragment $P_{S C O 4848}+S C O 4849$ was obtained in three steps: first, the promoter region of SCO4848 $\left(P_{S C O 4848}\right)$ was amplified from the $S$. coelicolor genome using primers $P_{S C O 4848} \mathrm{~F} / \mathrm{R}$ (incorporating HindIII and NdeI restriction sites at the $5^{\prime}$ and $3^{\prime}$ ends, respectively) and cloned into pCR $^{\text {TM}}$-Blunt II-TOPO ${ }^{\circledR}$ (pTOPO-P ${ }_{\mathrm{SCO} 4848}$ ); secondly, the ORF of SCO4849 was amplified with primers SCO4849 F/R (incorporating NdeI and NsiI restriction sites at the $5^{\prime}$ and $3^{\prime}$ ends, respectively) and cloned into pCR $^{\mathrm{TM}}$-Blunt II$\mathrm{TOPO}^{\circledR}$ selecting for the plasmid in which the $5^{\prime}$ end of the SCO4849 gene incorporating the NdeI restriction site was orientated to the HindIII side of the pCR ${ }^{\mathrm{TM}}$-Blunt IITOPO $^{\circledR}$ (pTOPO-SCO4849); thirdly, $P_{S C O 4848}$ was released from pTOPO-P $\mathrm{SCO}_{448}$ using HindIII and NdeI and cloned 


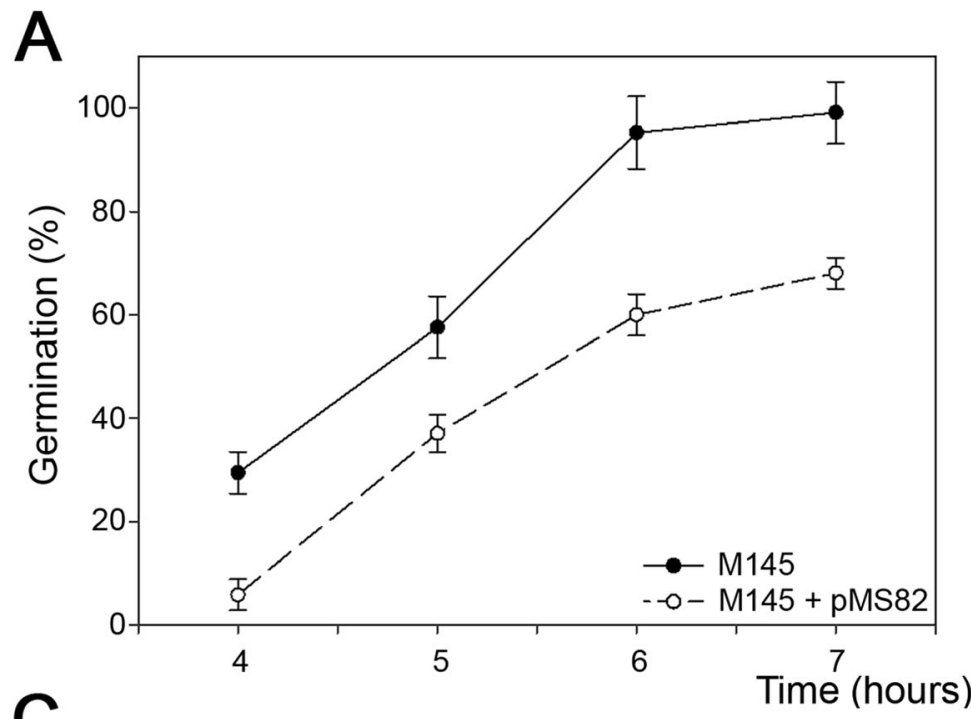

S. coelicolor

S. coelicolor chromosome

B

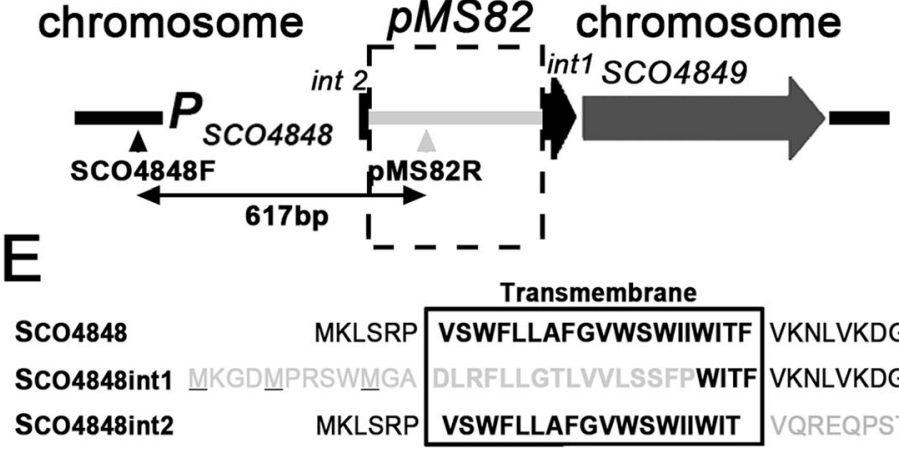

Transmembrane

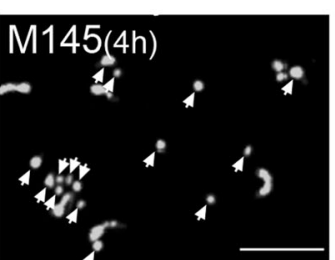

M145+pMS82(4h)
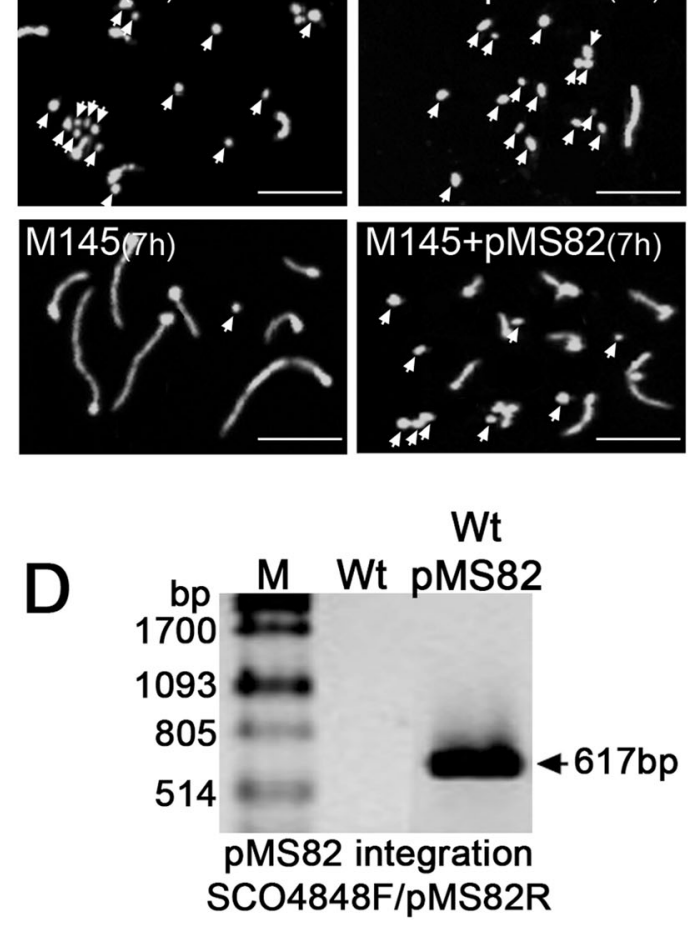

Transmembrane

SC04848 MKLSRP VSWFLLAFGVWSWIIWITF VKNLVKDGSGLAFEDGDPT AYFWVHLLLAIVSFVLGTVVGVIGL RGVRALRRTS SC04848int1 MKGD MPRSWMGA DLRFLLGTLVVLSSFP WITF VKNLVKDGSGLAFEDGDPT AYFWVHLLLAIVSFVLGTVVGVIGL RGVRALRRTS Sc04848int? MKLSRP VSWFLLAFGVWSWIIWIT

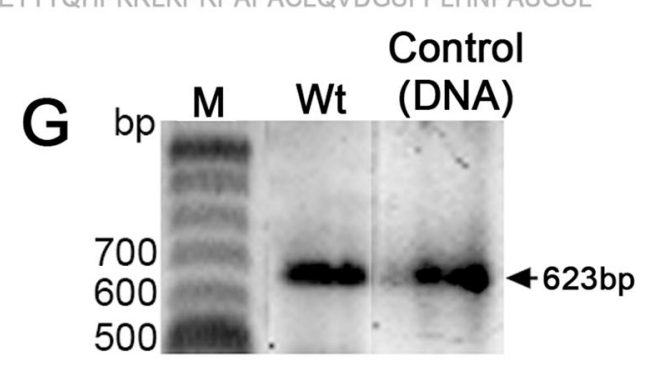

$\mathrm{F}$

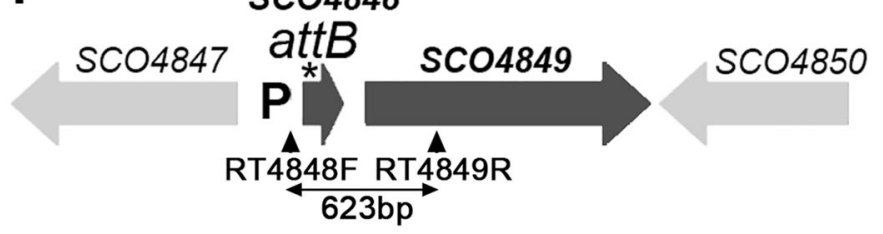

$\mathrm{H}$

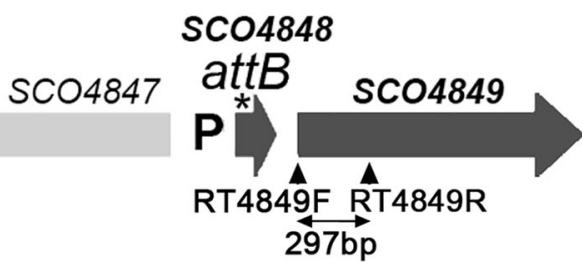

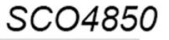

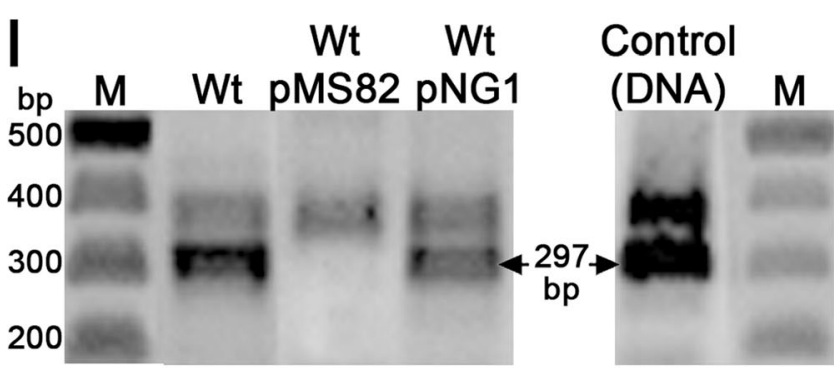

into pTOPO-SCO4849 digested with the same enzymes, generating pTOPO- $\mathrm{P}_{\mathrm{SCO} 4848}+\mathrm{SCO} 4849$. Fragment $P_{S C O 4848}+S C O 4849$ was released from pTOPO-P ${ }_{\mathrm{SCO} 4848}{ }^{+}$ SCO4849 using HindIII and NsiI, and it was religated into
pMS82 (Gregory et al. 2003) digested with the same enzymes, generating pNG1 (accession number KR131848). pMS82 containing $P_{S C O 4848}+S C O 4848+S C O 4849$ was constructed by cloning the synthetized SCO4848 gene 
(accession number KR150757) harbouring synonymous mutations (Fig. 1d) into pNG1 (both digested with NdeI). As detailed below, this construction was unstable.

\section{pNG2 construction}

pNG1 was digested with NdeI. NdeI single-stranded overhangs were digested with nuclease S1 (Thermo Scientific), and the plasmid was religated using T4 DNA ligase (Invitrogen) to generate plasmid pNG1' without the NdeI restriction site. The synthetized fragment $P_{\text {ermE }}{ }^{*}+\mathrm{RBS}+$ MCS+fd-ter, digested with EcoRV-XbaI, was cloned into pNG1' digested with EcoRV-SpeI ( $X b a \mathrm{I}$ and SpeI are compatible enzymes) generating pNG2 (accession number KR131849).

\section{pNG3 construction}

The bla gene conferring resistance to ampicillin was amplified from plasmid pBR322 (Fermentas) using primers Bla-F and Bla-R (Table 1) and Phusion High-Fidelity DNA Polymerase (Thermo). The amplified gene was cloned into $\mathrm{pCR}^{\mathrm{TM}}-\mathrm{Blunt}$ II-TOPO ${ }^{\circledR}$. The bla gene was released from $\mathrm{pCR}^{\mathrm{TM}}$-Blunt IITOPO with HindIII/AvrII and cloned into pNG1 digested with the same enzymes, generating pNG3 (accession number KR131850).

\section{pNG4 construction}

The bla gene was released from pCR ${ }^{\mathrm{TM}}$-Blunt II-TOPO with HindIII/AvrII and cloned into pNG2 digested with the same enzymes, generating pNG4 (accession KR131851).

\section{Disruption and overexpression of SCO0995}

The transposon insertion single-gene knockout library created by Prof. P. Dyson's research group (Fernández-Martínez et al. 2011) was used for mutagenesis of SCO0995. Cosmid 2StG2.1.G12 was used for constructing the SCO0995 mutant strain $(\triangle \mathrm{SCO} 0995)$ with the SCO0995 gene interrupted by the Tn5062 transposon (Table 1). Gene disruption was carried out by obtaining double crossovers via conjugation using E. coli ET12567/pUZ8002 as a donor strain and following the protocol described in Kieser et al. (2000). Mutant strains were confirmed by Southern blotting, using chromosomal DNA digested with SalI. Southern hybridization was carried out by established procedures using the digoxigenin-labelled 3442-bp Tn5062 PvuII fragment from plasmid pQM5062 (Bishop et al. 2004) as a probe.

The ORF of SCO0995 gene was amplified from $S$. coelicolor genome using primers $\mathrm{SCO} 0995 \mathrm{~F}$ and SCO0995R primers and cloned in pNG2 (into the NdeI-SpeI restriction sites) under the control of PermE*.

\section{Bioinformatic analyses}

Transmembrane topology of the SCO4848 and SCO4849 genes was analysed by Phobius software (http://phobius.sbc. su.se/). The rest of the bioinformatics and phylogenetic analyses were performed using the Phylemon 2.0 bioinformatic tools (Sánchez et al. 2011).

Orthologous sequences to SCO4848 and SCO4849 from other streptomycetes were obtained from the databases at the National Center for Biological Information (http://www.ncbi. nlm.nih.gov). SCO4848 was not annotated in the genomes of S. lividans or S. griseus; however, the ORF was present in their respective genomes (StrepDB database). The sequences selected were SLI 5122 (S. lividans), SAV 3412 and SAV 3411 (S. avermitilis); SVEN_4521 and SVEN_4522 (S. venezuelae); SCAB35521, SCABB35511 (S. scabies), SGR 2696 (S. griseus) and SCLAV_3766, SCLAV_3767 (S. clavuligerus). Amino acid sequences were aligned using MUSCLE software, and amino acid similarities were estimated by Lalign software (http://www.ch.embnet.org/ software/LALIGN_form.html).

\section{Results}

\section{Phenotype caused by the integration of pMS82 into the $S$. coelicolor genome}

Key phenotypes of the $S$. coelicolor strain harbouring pMS82 were analysed and compared with $S$. coelicolor wild-type strain. Spore germination, first compartmentalized mycelium, second multinucleated mycelium without (substrate) or with (aerial) hydrophobic covers, and sporulation were analysed in solid cultures (Supplementary Figures S1, S2). Antibiotic production (undecylprodigiosin and actinorhodin) was analysed in liquid cultures (Supplementary Figure S3). As discussed below, the only phenotypic difference observed in the S. coelicolor harbouring pMS82, with respect to the wildtype strain, was a delay in spore germination (Fig. 1a, b).

Spore germination timing depends on the culture medium used for germination (GYM, R2YE or MM) (Fig. 1a and Supplementary Figure S4). However, the delay in germination generated by the integration of $\Phi \mathrm{BT} 1$ plasmids was observed in all the strains and all the media tested.

The effect of the medium used to raise the spores was tested in S. coelicolor, comparing the differences in germination of spores obtained from SFM or GYM (Fig. 1a and Supplementary Figure S4). In both cases, pMS82 reduced spore germination. Differences in the percentage of germination between the two batches of spores were not significant. It is notable that germination in $S$. coelicolor is very fast (starting at $4 \mathrm{~h}$ and finishing at around $7 \mathrm{~h}$ ) (Fig. 1a), and small variations in the cultures can generate differences in the exact 
percentage of germination observed at a specific developmental time point.

\section{\$BT1 integration in $S$. coelicolor chromosome}

The integration site of $\Phi \mathrm{BT} 1$ plasmids into the S. coelicolor genome was reported to be in the SCO4848 gene (Gregory et al. 2003). To our knowledge, there are no studies describing the existence of alternative $\Phi B T 1-a t t B$ sites in Streptomyces, which is in contrast to the sites for $\Phi C 31$ integration (Combes et al. 2002). Two primers were designed in this work, hybridizing at the SCO4848 $5^{\prime}$ end and the DNA region flanking attP in pMS82 (Fig. 1c). These primers amplify a fragment of 617 bp only if pMS82 or their derived plasmids are integrated at the $a t t B$ site of SCO4848 (Figs. 1d). The pMS82- and the pMS82-based plasmids created in this work (pNG1-pNG4, see below) were used in our lab in 24 independent experiments to complement wild phenotypes in different $S$. coelicolor mutants, and in all of them, the integration was found at the $S C O 4848$ attB site (Supplementary Figure S5). This suggests that $S C O 4848$ harbours the only $\Phi B T 1$-att $B$ site in $S$. coelicolor, which, as discussed below, is one of the key requirements to construct a phenotypically neutral integrative plasmid in Streptomyces.

Integration of the $\Phi \mathrm{BT} 1$ plasmid interrupts $S C O 4848$ by modifying its $5^{\prime}$ end. Integration generates a modified SCO4848 ORF (SCO4848int1 in Fig. 1e), which retains a putative signal peptide, and the key features of SCO4848 and was predicted to be functional (Gregory et al. 2003). However, as discussed below, the SCO4848int1 gene and the promoter region of $S C O 4848$ are separated by the integrated plasmid, and the transcription of SCO4848int1 is very unlikely (Fig. 1c). SCO4849 is located downstream of SCO4848 (Fig. 1f), and RT-PCR experiments performed in this work demonstrated that SCO4848 is co-transcribed with SCO4849 in the S. coelicolor wild-type strain (Fig. 1g). Thus, the integration of $\Phi \mathrm{BT} 1$ plasmids into SCO4848 might also affect the expression of SCO4849. The alteration of SCO4849 transcription was confirmed by RT-PCR using primers RT4849F and RT4849R (Fig. 1h, i). This RT-PCR amplified more than one amplicon; however, the expected amplicon (297 bp) was present in S. coelicolor wild type, and it was absent in the strain harbouring pMS82 (Fig. 1i). As stated in "Materials and methods" section, the identity of this 297-bp amplicon was confirmed by DNA sequencing (data not shown).

\section{Construction of $\Phi B T 1$ integrative plasmids restoring the expression of $\mathrm{SCO} 4849$}

In order to create $\Phi \mathrm{BT} 1$ integrative plasmids restoring the expression of SCO4848 and SCO4849, after the plasmid had integrated into the Streptomyces genome, two genetic constructs were synthetized (Fig. 2a): the first one, containing a 423-bp fragment upstream of the ATG of the SCO4848 gene, large enough to encompass the promoter region of the $S C O 4848$ gene $\left(P_{S C O 4848}\right)$, together with the ORFs of SCO4848 and SCO4849 $\left(P_{S C O 4848}+S C O 4848+S C O 4849\right)$; and the second one, consisting of the promoter of SCO4848 and the ORF of SCO4849 $\left(P_{S C O 4848}+S C O 4849\right)$. In order to try to inactivate the attB site of $S C O 4848$ and to prevent the recombination with the attP site of the plasmid, the SCO4848 amino acid sequence was retained, but the nucleotides of the $a t t B$ site were changed by synonymous mutations (Fig. 2a).

$P_{S C O 4848}+S C O 4848+S C O 4849$ and $P_{S C O 4848}+S C O 4849$ were introduced into the $\Phi B T 1$ lasmid pMS82 (Gregory et al. 2003); however, only the second one $\left(P_{S C O 4848}+S C O 4849\right)$ was stable, generating plasmid pNG1 (Fig. 3a). Introduction of $P_{S C O 4848}+S C O 4848+S C O 4849$ into pMS82 generated plasmid reorganizations and deletions in pMS82 (Supplementary Figure S6), indicating that the mutated $a t t B$ site synthetized (Fig. 2b) was still functional after recombining with the $a t t P$ site of the plasmid.

The phenotype in spore germination generated by the integration of $\Phi \mathrm{BT} 1$ integrative plasmids into SCO4848 (see above and Fig. 1a, b) was complemented by the $P_{S C O 4848^{+}}$ SCO4849 fragment included in pNG1 (Fig. 2c), and the restauration of the SCO4849 expression in the S. coelicolor strain harbouring pNG1 was demonstrated by RT-PCR (Fig. 1i). These results demonstrated that the phenotype detected was a consequence of the alteration of SCO4849 expression due to the plasmid integration.

To facilitate work in Streptomyces, three more plasmids were constructed based on pNG1: pNG2, in which the strong constitutive promoter $P_{\text {ermE }}{ }^{*}$ (Bibb et al. 1994), the RBS of the gene tuf-1 (Vijgenboom et al. 1994), a cloning site including single-cutter restriction enzymes, and the terminator of the fd phage (Gentz et al. 1981) were introduced into pNG1 to facilitate heterologous gene expression and protein translation (Fig. 3b); pNG3, in which the bla gene for ampicillin resistance was included in pNG1 in order to facilitate selection in E. coli (Fig. 3c); and pNG4, in which the bla gene was introduced into pNG2 (Fig. 3d).

The functionality of the $P_{\text {ermE }}{ }^{*}$ and RBS site included in pNG2 and pNG4 was tested using pNG2 to overexpress the ORF of SCO0995 (plasmid pNG2-SCO0995) (Table 1), encoding a putative DNA-methyltransferase, into a $\triangle$ SCO0995 strain (mutated in SCO0995) (Fig. 4a). The integration of the pNG2-SCO0995 plasmid and the presence/ absence of the SCO0995 in the S. coeliocolor wild-type strain, the $\triangle \mathrm{SCO} 0995$ mutant and the mutant strain harbouring pNG2-SCO0995 were confirmed by PCR (Figs. 4b, c). As expected, the SCO0995 mRNA was detected in the wildtype and the mutant strain complemented with plasmid pNG2-SCO0995, but not in the $\triangle \mathrm{SCO} 0995$ mutant strain (Fig. 4e). 
A

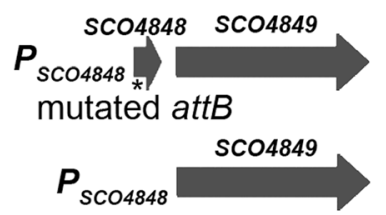

C

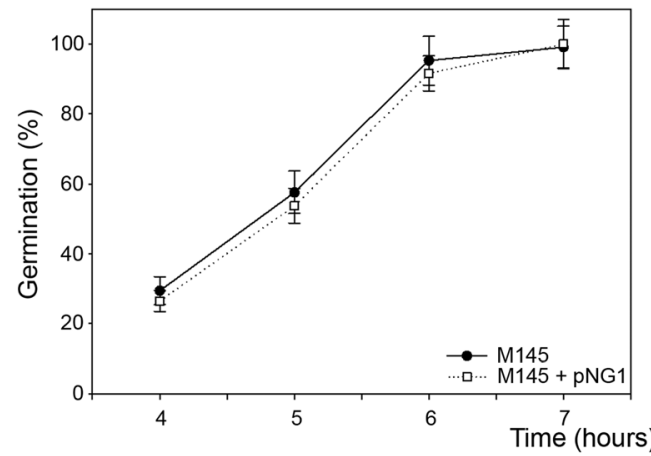

Fig. 2 Construction of $\Phi B T 1$ integrative plasmids restoring the expression of SCO4849. a Genetic constructs to restore the expression of SCO4848 and SCO4849. The attB integrative site is indicated by an asterisk. b Detail of the sequence of the $a t t B$ and the mutated att $B$ harbouring synonymous mutations. c Percentage of germination
B

attB

CTC GCG TTC GGG GTG WGG AGC TGG ATC ATC TGG ATC

ACT TTC GTC AAA AAC CTG GTC AAG GACGGC AGCGGG C

mutated attB

CTIg GCC TFt GGC GTC WTG AS W W AT AT TWG ATt

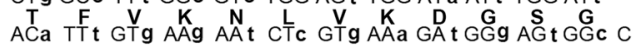

D

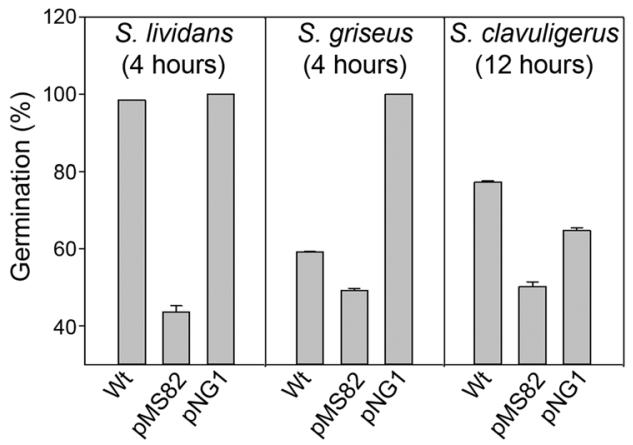

(average $\pm \mathrm{SD}$ ) in $S$. coelicolor M145 wild-type strain and $S$. coelicolor harbouring pNG1 growing in GYM medium. d Percentage of spore germination (average \pm SD) in $S$. griseus, $S$. lividans and S. clavuligerus with and without pMS82 or pNG1. GYM was used in the case of $S$. griseus and S. lividans; TSA was used for S. clavuligerus

\section{Discussion}

The $\Phi C 31$ integrative plasmids are widely used in streptomycetes; however, they have severe limitations due to the existence of several integration sites in the Streptomyces genome, which cause different mutations resulting in different phenotypes (Bierman et al. 1992). The most common alternative in Streptomyces to $\Phi \mathrm{C} 31$ integrative plasmids are the $\Phi \mathrm{BT} 1$-derived vectors which integrate at the unique $a t t B$ site localized in the ORF of $S C O 4848$ ( $S$. coelicolor genome). Mutation of $S C O 4848$ was reported to produce an ORF that remained functional (SCO4848int1 in Fig. 1e) and to be neutral in terms of phenotype (Gregory et al. 2003). However, the SCO4848int1 gene and the promoter region of SCO4848 are separated by the integrated plasmid (Fig. 1c), and the transcription of SCO4848int1 is very unlikely. The other ORF generated by the integration of the $\Phi \mathrm{BT} 1$ plasmids (SCO4848int2) has $30 \%$ of the amino acids of SCO4848 protein (24 amino acids of a total of 74) (Fig. 1e) under the control of the SCO4848 promoter, and it might conserve part of the function of the SCO4848 protein. In this work, we further characterized the phenotype generated by the integration of $\Phi B T 1$ plasmids into the Streptomyces genome, discovering the existence of a previously uncharacterized phenotype during the germination stages consisting of a delay in spore germination. This phenotype was demonstrated to be a consequence of an effect on SCO4849 expression, which encodes a putative integral membrane protein that is co-transcribed together with $S C O 4848$. Further work is required to identify 


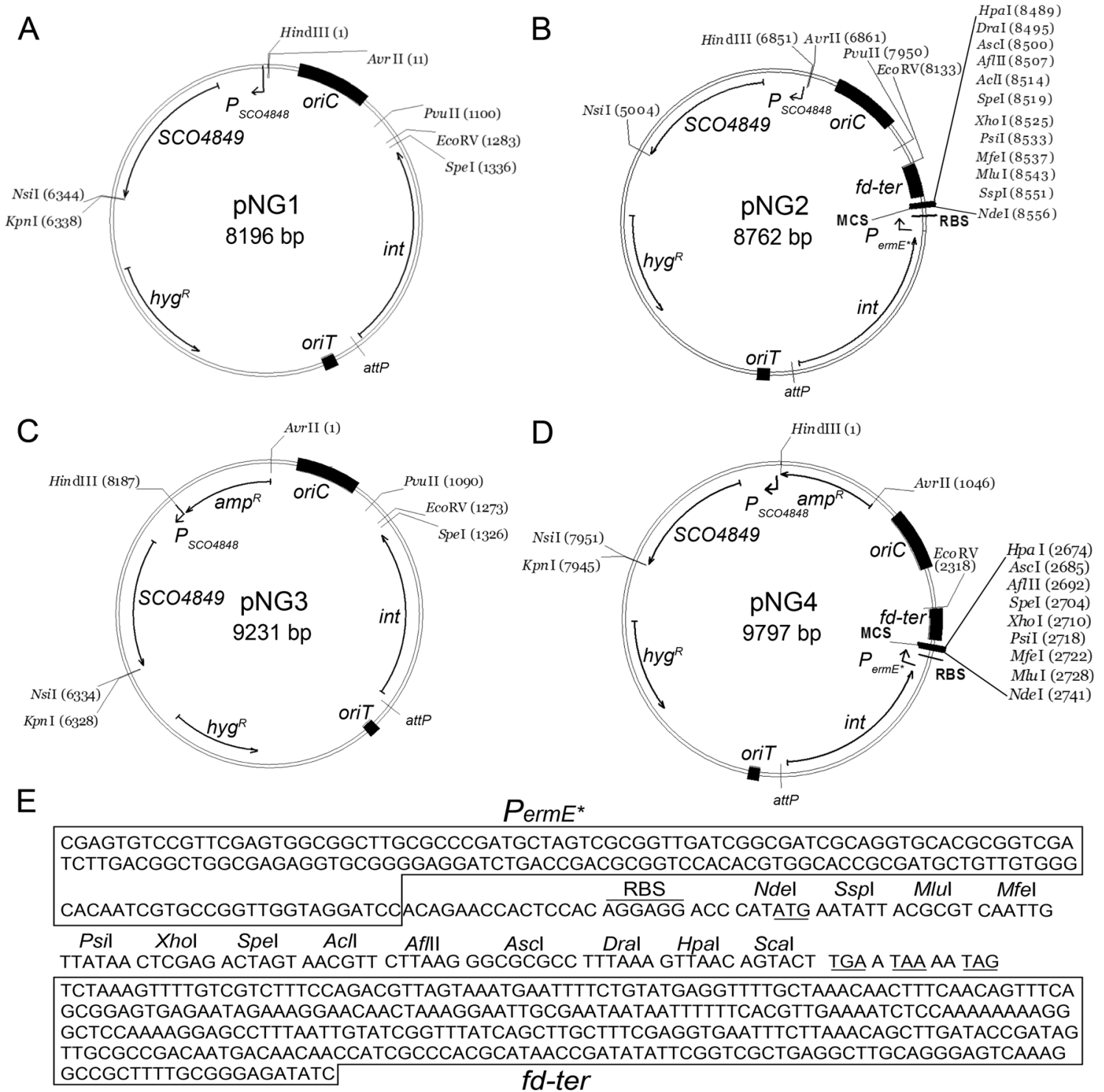

Fig. 3 QBT1 integrative plasmids pNG1, pNG2, pNG3 and pNG4. The basis of all these plasmids is pMS82 (Gregory et al. 2003). a pNG1 harbours $P_{S C O 4848}+S C O 4849$. b pNG2 harbours $P_{S C O 4848}+S C O 4849$ and $P_{\text {ermE }}{ }^{*}+\mathrm{RBS}+\mathrm{MCS}+$ fd-ter. c pNG3 harbours $P_{S C O 4848}+S C O 4849$ and bla (resistance to ampicillin). d pNG4 harbours $P_{S C O 4848}{ }^{+}$

the biological function, if any, of SCO4848, and to characterize the biochemical mechanism by which SCO4849 controls spore germination. Other phenotypes, such as antibiotic production, growth rate or sporulation, were not significantly affected by the integration of $\Phi B T 1$ vectors, consistent with the results previously reported by Gregory et al. (2003).

The aim of this work was the construction of phenotypically neutral integrative plasmids for use in Streptomyces. These plasmids needed to meet two main characteristics: first, they should have a unique and conserved integrative site in streptomycetes; and second, the mutation generated by the integration of the plasmid should not generate any phenotype.
SCO4849, $P_{\text {ermE }}{ }^{*}+\mathrm{RBS}+\mathrm{MCS}+f d$-ter and bla. e Sequence of the $P_{\text {ermE }}{ }^{*}+\mathrm{RBS}+\mathrm{MCS}+f d$-ter region. oriC origin of replication, Int $\Phi \mathrm{BT} 1$ integrase, oriT origin of transfer, hyg hygromycin resistance gene. Single-cutter enzymes are shown

Concerning the existence of a unique integrative site, ФBT1 integrative plasmids represent a good alternative (Gregory et al. 2003). To our knowledge, no studies are available of the existence of alternative $\Phi B T 1-a t t B$ sites different from the $a t t B$ site located at SCO4848. In this work, it was demonstrated in 24 independent integrations (Supplementary Figure S5) that pMS82 and their derived plasmids were always integrated into SCO4848, suggesting that $\mathrm{SCO} 4848$ harbours a unique $\Phi \mathrm{BT} 1-$ att $B$ site in $S$. coelicolor. In fact, a BLAST of the SCO4848 attB sequence against the $S$. coelicolor genome did not retrieve alternative putative attB sites (data not shown). 


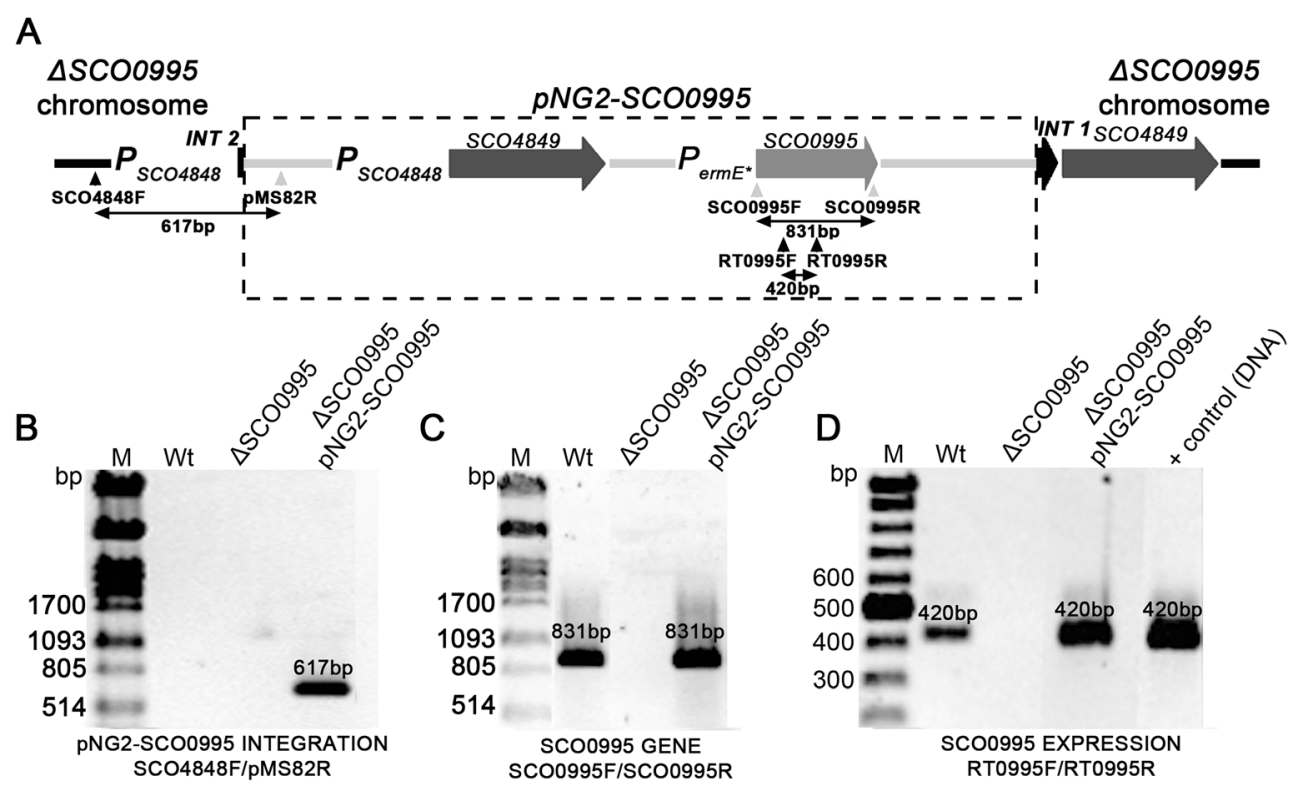

Fig. 4 Functionality of the expression vector pNG2 to restore SCO0995 expression in the $\Delta \mathrm{SCO} 0995$ mutant. a Scheme illustrating the integration of pNG2-SCO0995 into the $\triangle \mathrm{SCO} 0995$ chromosome. b PCR using primers SCO4848F and pMS82R confirming plasmid integration into SCO4848. c PCR using primers SCO0995F and

SCO0995R confirming the presence of the SCO0995 gene into the $\triangle \mathrm{SCO} 0995$ strain harbouring pNG2-SCO0995. d RT-PCR demonstrating the functionality of the expression vector $\mathrm{pNG} 2$. SCO0995 is expressed in the SCO0995 mutant strain ( $\triangle$ SCO0995) harbouring the pNG2-SCO0995 plasmid

Fulfilling the second requirement, the absence of a phenotype after plasmid integration is more difficult to achieve because general integrative sites need to be conserved, and conserved genes are usually important, and their mutations lead to phenotypes. As discussed above, the integration of $\Phi \mathrm{BT} 1$ plasmids into the $S$. coelicolor genome generates a spore germination phenotype. The most obvious approach to construct a phenotypically neutral integrative plasmid is to introduce the chromosomal gene/s interrupted by the integration, into the integrative plasmid. However, this was not possible in the case of pMS82 due to the interaction between the attP site of the plasmid and the attB site of the gene to be complemented, which made these plasmids unstable (Supplementary Figure S6). Fortunately, the phenotype detected during germination after the integration of $\Phi \mathrm{BT} 1$ plasmids was a consequence of the absence of the gene located downstream of the attB integration site (SCO4849 in the case of S. coelicolor). This allowed us to construct a $\Phi \mathrm{BT} 1$ integrative plasmid including the promoter of the $S C O 4848$ gene fused with the ORF of SCO4849 (pNG1), which complements the phenotype in germination generated by the integration of the plasmid into SCO4848. pNG1 is in fact the first integrative plasmid in $S$. coelicolor demonstrated to be phenotypically neutral. The $P_{\text {ermE }}{ }^{*}$ promoter, a RBS, a multiple cloning site and the terminator of the fd phage (fd-ter) were introduced in pNG1 to facilitate heterologous gene expression, creating pNG2. Both plasmids showed a resistance to hygromycin which is effective in Streptomyces. In E. coli, however, false resistant colonies can grow, albeit at a slower rate than the E. coli harbouring the plasmids with the resistance, making hygromycin selection challenging. For this reason, the bla gene coding for ampicillin resistance was included into pNG1 and pNG2 in order to facilitate selection in E. coli, thus generating plasmids pNG3 and pNG4.

SCO4848 and SCO4849 orthologues are highly conserved in streptomycetes (Fig. 5), and the delay in spore germination as a consequence of the integration of $\Phi \mathrm{BT} 1$ plasmids into SCO4848 and their orthologues, may be common in Streptomyces (Fig. 2c, d). pNG1-4 are useful in other streptomycetes beyond $S$. cocelicolor; however, the usefulness of these plasmids requires verification for each specific Streptomyces species because they do not always complement the wild phenotypes. For instance, pNG1 accelerates the germination in S. griseus with respect to the wild-type strain, indicating that the effect of the $S$. coelicolor SCO4849 is stronger than that of the $S$. griseus orthologue mutated by the integration of the plasmid. In these types of strains, alternative SBT1 plasmids harbouring the SCO4849 orthologue of the specific host strain might be created and tested.

Overall, this work describes the construction of plasmids pNG1-4, the first integrative vectors designed to restore the expression of the genes interrupted by their integrations, producing neutral phenotypes in S. coelicolor, S. lividans and $S$. clavuligerus. These vectors facilitate the interpretation of the phenotypes generated by the integration of heterologous DNA, limiting the interference of the phenotypes caused by the integration vectors. The experimental approach developed in this work to create phenotypically neutral $\Phi B T 1$ integrative plasmids in $S$. coelicolor might be applied to other bacterial integrative vectors. 
B

Transmembrane $(86 \%$ average similarity)

\section{SCO4849 ORTHOLOGUES}

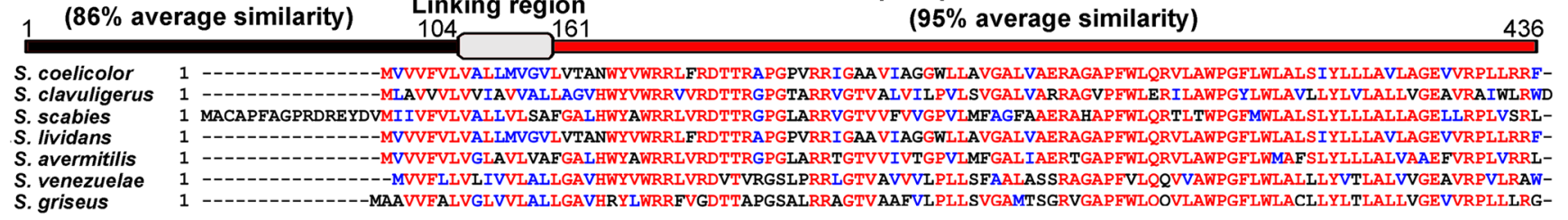

S. coelicolor 104 -IERPAAARR-S. clavuligerus 105 RTAKRTARSGRPETTATAAPVTSTTTSTITGTTTSTTTSSATGADAGTAPVAARPEPVPVPGAEEPRETAARQDTDAGTASASGTSSGTAKPEPAPESEPASEPVTEIAPAPGPAAAEPA S. scabies 120 -VARRAPAPAPRPE PAEREP---------PSATEPPPVAPSA S. lividans 104 -LERRAAARR--S. avermitilis 104 -VEREAPAEVAVPQPER--------------------------VPAGTAGTAATE------------------PEPEVESAPEPKRPEP--------AGP-------PRA S. venezuelae 103 -LTRRAHR--

S. griseus 105 -LARRDGRPGTAESGTASGAPAGLPGEPRPLAGELVPSGSAGAPTRSEAVTRTAADPVTEPETE--------------PTATAPATSAEAPVPAAVAE----------APAPATDAQAQA

S. coelicolor 161 AVPSRRLFVSRVVAGAAAAAAVGTVGYGTYGVLSGPKVKRVTVPLAKLPRAAHGFRIAVVSDIHLGPVLGRGFAQQVVDTINSTQPDLIAVVGDLVDGSVKDLGPAAAPLARLTARHGAY S. clavuligerus 225 RAVSRRLFVSRTVAAGAAAAALGTVGHGAHGVLRGPRLKRVTVPLAKLPRSAHGLRIAVVSDIHIGPILGRAHTQRIVDTINAAQPDLVAVVGDMVDGTVADLGPAAEPLAQLRARHGSF S. clavies 207 ADPSRRLFVSRVVGGAVAAAAVGTVGYGTYGVVRGPRLKRVTVPLAKLPRAAHGFRIAVVSDIHLSPMLGRGFAQKIVDTINSTQPDLIAVVGDLVDGDVADLGPAAAPLAGLKARHGSY
S. scabies S. lividans 161 AVPSRRLFVSRVVAGAAAAAAVGTVGYGTYGVLSGPKVKRVTVPLAKLPRAAHGFRIAVVSDIHLGPVLGRGFAQQVVDTINSTQPDLIAVVGDLVDGSVKDLGPAAAPLARLRARHGAY S. avermitilis 154 AAPSRRLFVSRVVGGAAAAAAVGTVGYGTYGVLRGPRVKRVTVPLARLPRSAHGFRIAVVSDIHLSPVLGRGFAQRVVDTINSTQPDLIAVVGDLVDGSVQDLGPAAAPLSQLKARHGSY S. venezuelae 133 SDVSRRLFVSRVVGGAAAAAAVGTVGVGTYGVLRGPRIKRVTVPLAKVPRAAHGYRIAVVSDIHLGPILGRAHTQRIVDTLNSAQPDLIAVVGDLVDGTVENLGSAAEPLARLRARHGSF S. griseus 200 PGPSRLFVSRVVGGAAAAAGLATVGYGTYGVLRGPSVRRVTVPLAKLPRSAHGFRIAVVSDIHIGPILGRAHTRRIVDTINATSPDLVAVVGDLVDGSVADLGSAAEPLAALRARHGSY

S. coelicolor 281 FVTGNHEYFSGAEQWVAEVRRLGLLPLENARTELP-HFDLAGVNDVAGEDEGQGPDYDRALGDRDRSRACVLLAHQPVQIHDAVDHGVDLQLSGHTHGGQLWPGNLIAGAANPTLAGLER S. clavuligerus 345 FVTGNHEYFSGAEQWVEHVRELGLKPLENERVEIAGGFDLAGVNDVEGESTGQGPDFARALGDRDRSRAAVLIAHQPVVIHDAVEHGVDLQLSGHTHGGQLWPGNYLAELANPTVAGLER S. scabies 327 FVTGNHEYISGAGQWVEEVRRLGLTPLENARRELP-YLDLAGVNDIAGEDEGQGPDFAKALGDRDTSRAVVLMAHQPVQIHDAVDHGVDLQLSGHTHGGQLWPMTYVAQAANPTLAGLER S. lividans 281 FVTGNHEYF GAEQWVAEVRRLGLLPLENARTELP-HFDLAGVNDVAGEDEGQGPDYDRALGDRDRSRACVLLAHQPVQIHDAVDHGVDLQLSGHTHGGQLWPGNLIAGAANPTLAGLER S. avermitilis 274 FVTGNHEYFSGAGQWVEEVRRLGLNPLENARTELP-AFDLAGVNDVAGESEGQGPDFDRALGDRDTTRAVVLLAHQPVQIHEAVRHDVDLQLSGHTHGGQLWPGSLVAAAANPTVAGLER S. venezuelae 253 FVTGNHEYFSGADAWVDHVRELGLRPLRNDRVEIAAGFDLAGVDDVAGESEGQGPDFVRALGDRDRARAAVLLAHQPIVVHDAVRHGVDLQLSGHTHGGQLWPGNFLAELANPTVAGLER S. griseus 320 FVTGNHEYF SGEQWVDHVRELGLIPLENARVEIG-GFDLAGVNDIAGETEGQGPDFGRALGDRDRGRAAVLLAHQPVVIHDAVEHGVDLQLSGHTHGGQLWPGNLLAGLANPTVDGLER

S. coelicolor 400 YGDTQLYVSRGAGAWGPPTRVGAPSDITVIELASRQA

S. clavuligerus 465 YGDTQLYVSRGAGAWGPPVRVGAESDITIVQLASRQA

S. scabies 446 YGDTQLYVSRGAGAWGPPVRVGAPSDITVVQLASKQA

S. lividans 400 YGDTQLYVSRGAGAWGPPTRVGAPSDITVIELASRQA

S. avermitilis 393 YGDTQLYVSRGAGAWGPPTRVGAPSDITVVELASKQA

S. venezuelae 373 YGDTQLYVSRGAGAWGPPVRVGAPSDITIVELASKQA

Fig. 5 Sequence alignment of SCO4848 and SCO4849 (S. coelicolor numeration) and their orthologues in other model streptomycetes. a SCO4848 orthologues. b SCO4849 orthologues. Putative

Acknowledgments Our thanks to Maggie Smith (University of York) for providing the pMS82 plasmid, Beatriz Gutiérrez Magán (Universidad de Oviedo, Dpto. Biología Funcional, Área de Microbiología) for the laboratory assistance, and Proof-Reading-Service.com for proofreading the text.

Compliance with ethical standards

\section{Ethical statement}

Funding This research was funded by an ERC Starting Grant (Strp-differentiation 280304). NGQ was funded by a Severo Ochoa fellowship (FICYT, Consejería de Educación y Ciencia, Spain).

Conflict of interest The authors declare that they have no competing interests.

Ethical approval This article does not contain any studies with human participants or animals performed by any of the authors. transmembrane regions, average similarities and conserved database domain references $(C D D)$ are indicated

\section{References}

Bibb MJ, White J, Ward JM, Janssen GR (1994) The mRNA for the 23S rRNA methylase encoded by the ermE gene of Saccharopolyspora erythraea is translated in the absence of a conventional ribosomebinding site. Mol Microbiol 14:533-545

Bierman M, Logan R, O’Brien K, Seno ET, Rao RN, Schoner BE (1992) Plasmid cloning vectors for the conjugal transfer of DNA from Escherichia coli to Streptomyces spp. Gene 116:43-49

Bishop A, Fielding S, Dyson P, Herron P (2004) Systematic insertional mutagenesis of a streptomycete genome: a link between osmoadaptation and antibiotic production. Genome Res 14:893-900

Bradford MM (1976) A rapid and sensitive method for the quantitation of microgram quantities of protein utilizing the principle of protein-dye binding. Anal Biochem 72:248-254

Bystrykh LV, Fernandez-Moreno MA, Herrema JK, Malpartida F, Hopwood DA, Dijkhuizen L (1996) Production of actinorhodinrelated "blue pigments" by Streptomyces coelicolor A3(2). J Bacteriol 178:2238-2244 
Combes P, Till R, Bee S, Smith MC (2002) The Streptomyces genome contains multiple pseudo-attB sites for the (phi)C31-encoded sitespecific recombination system. J Bacteriol 184:5746-5752

Davies J (2014) Specialized microbial metabolites: functions and origins. J Antibiot (Tokyo) 66:361-364

Fayed B, Younger E, Taylor G, Smith MC (2014) A novel Streptomyces spp. Integration vector derived from the $S$. venezuelae phage, SV1. BMC Biotechnol 14:51

Fernandez E, Weissbach U, Sanchez-Reillo C, Braña AF, Mendez C, Rohr J, Salas JA (1998) Identification of two genes from Streptomyces argillaceus encoding glycosyltransferases involved in transfer of a disaccharide during biosynthesis of the antitumor drug mithramycin. J Bacteriol 180:4929-4937

Fernández-Martínez LT, Del Sol R, Evans MC, Fielding S, Herron PR, Chandra G, Dyson PJ (2011) A transposon insertion single-gene knockout library and new ordered cosmid library for the model organism Streptomyces coelicolor A3(2). Antonie Van Leeuwenhoek 99:515-522

Flett F, Mersinias V, Smith CP (1997) High efficiency intergeneric conjugal transfer of plasmid DNA from Escherichia coli to methyl DNA-restricting streptomycetes. FEMS Microbiol Lett 155:223229

Gentz R, Langner A, Chang AC, Cohen SN, Bujard H (1981) Cloning and analysis of strong promoters is made possible by the downstream placement of a RNA termination signal. Proc Natl Acad Sci U S A 78:4936-4940

Gregory MA, Till R, Smith MC (2003) Integration site for Streptomyces phage phiBT1 and development of site-specific integrating vectors. J Bacteriol 185:5320-5323

Hopwood DA (2007) Streptomyces in nature and medicine: the antibiotic makers. Oxford University Press, New York

de Jong W, Manteca A, Sanchez J, Bucca G, Smith CP, Dijkhuizen L, Claessen D, Wösten HA (2009) NepA is a structural cell wall protein involved in maintenance of spore dormancy in Streptomyces coelicolor. Mol Microbiol 71:1591-1603
Kieser T, Bibb MJ, Buttner MJ, Chater KF, Hopwood DA (2000) Growth and preservation of Streptomyces. In Practical Streptomyces genetics, Chapter 2, pp. 43-61. Norwich, UK: The John Innes Foundation

MacNeil DJ, Gewain KM, Ruby CL, Dezeny G, Gibbons PH, MacNeil T (1992) Analysis of Streptomyces avermitilis genes required for avermectin biosynthesis utilizing a novel integration vector. Gene 111:61-68

Manteca A, Fernandez M, Sanchez J (2006) Cytological and biochemical evidence for an early cell dismantling event in surface cultures of Streptomyces antibioticus. Res Microbiol 157:143-152

Morita K, Yamamoto T, Fusada N, Komatsu M, Ikeda H, Hirano N, Takahashi H (2009) The site-specific recombination system of actinophage TG1. FEMS Microbiol Lett 297:234-240

Novella IS, Barbes C, Sánchez J (1992) Sporulation of Streptomyces antibioticus ETHZ 7451 in liquid culture. Can J Microbiol 38: 769-773

Sambrook J, Russell D (2001) Molecular cloning: a laboratory manual, Ed 3. Cold spring harbour laboratory press. Cold Spring Harbour, $\mathrm{NY}$

Sánchez L, Braña AF (1996) Cell density influences antibiotic biosynthesis in Streptomyces clavuligerus. Microbiology 142:1209-1220

Sánchez R, Serra F, Tárraga J, Medina I, Carbonell J, Pulido L, de María A, Capella-Gutíerrez S, Huerta-Cepas J, Gabaldón T, Dopazo J, Dopazo H (2011) Phylemon 2.0: a suite of web-tools for molecular evolution, phylogenetics, phylogenomics and hypotheses testing. Nucleic Acids Res 39:470-474

Tsao SW, Rudd BA, He XG, Chang CJ, Floss HG (1985) Identification of a red pigment from Streptomyces coelicolor A3(2) as a mixture of prodigiosin derivatives. J Antibiot (Tokyo) 38:128-131

Vijgenboom E, Woudt LP, Heinstra PW, Rietveld K, van Haarlem J, van Wezel GP, Shochat S, Bosch L (1994) Three tuf-like genes in the kirromycin producer Streptomyces ramocissimus. Microbiology 140:983-998

Yagüe P, López-García MT, Rioseras B, Sánchez J, Manteca A (2013) Pre-sporulation stages of Streptomyces differentiation, state-of-theart and future perspectives. FEMS Microbiol Lett 342:79-88 